University of Wollongong

Research Online

Faculty of Informatics - Papers (Archive)

Faculty of Engineering and Information

Sciences

1978

\title{
A class of codes generated by circulant weighing matrices
}

K Wehrhahn

Jennifer Seberry

University of Wollongong, jennie@uow.edu.au

Follow this and additional works at: https://ro.uow.edu.au/infopapers

Part of the Physical Sciences and Mathematics Commons

\section{Recommended Citation}

Wehrhahn, K and Seberry, Jennifer: A class of codes generated by circulant weighing matrices 1978 . https://ro.uow.edu.au/infopapers/989 


\title{
A class of codes generated by circulant weighing matrices
}

\author{
Abstract \\ Some properties of a new class of codes constructed using circulant matrices over GF(3) will be \\ discussed. In particular we determine the weight distributions of the $(14,7)$ and two inequivalent $(26,13)$ - \\ codes arising from the incidence matrices of projective planes of orders 2 and 3. \\ Disciplines \\ Physical Sciences and Mathematics \\ Publication Details \\ Wehrhahn, K and Seberry, J, A class of codes generated by circulant weighing matrices, Combinatorial \\ Mathematics: Proceedings of the international Conference, Canberra, August, 1977, 686, in Lecture Notes \\ in Mathematics, Springer--Verlag, Berlin--Heidelberg--New York, 1978, 282-289.
}


Licliure Noted in Mathanatios, Vol.686, Sprenger - Verlag, Burlin-Hecicitbery- Dlew York, pp 282-289 (1978). $\because \quad \begin{aligned} & 94 A 20 \\ & (05 B 20,05 B 30)\end{aligned}$

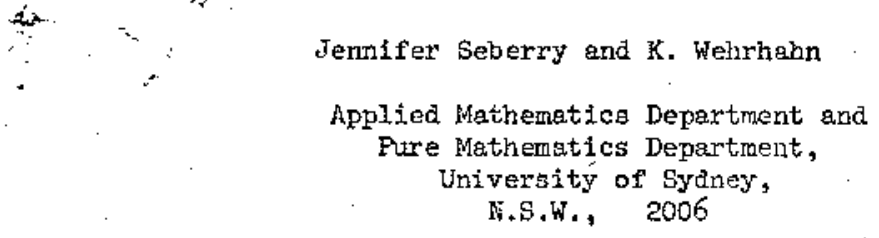

ABSFRACT.

Some properties of a new class of codes constructed using circulant matrices over GP(3) will be discussed. In perticutar we determine the weight distributions of the $(14,7)$ and two inequivalet $(26,13)$-codes arising from the incidence natrices of projective planes of orders 2 and 3.

1. INFHODUCTION.

In this paper "code" will meen a linear code over GF(3). An $(n, k)$-code $C$ has Iength $n$, dimension $k$. An. $(n, k, d)$-code is an $(n, k)-c o d e$ with minimum non-zero weight a. Oru notation and definitions are consistant with those of Blake and Mullin [2].

Let $Q$ be the circulant j.ncidence matrix of a projective plare of order $q$ (See Hall [6]). Then $Q$, of order $q^{2}+q+1$ satisfies

$$
\mathrm{QQ}^{\mathrm{T}}=\mathrm{qI}+\mathrm{J}, \quad \mathrm{QJ}=(\mathrm{q}+\mathrm{I}) \mathrm{J}
$$

where $J$ is the appropriate all $1^{\dagger}$ s matrix. $t_{n}=Q^{2}-J$ is a clrcular $(0,1,-1)$ matrix of order $q^{2}+q+I$ satisfying

$$
W^{T}=q^{2} I, \quad \quad T^{T} N^{T}=q J
$$

i.e. $W$ is a eireutant weighing matrix of weicht $q^{2}$. We write $w=w\left(q^{2}+q^{+1}, q^{2}\right)$ to denote its order and weight. Wore details of $W$ can be found in Hain [5] and Wallis and whiteman [10].

We call goins with basis

$$
\begin{aligned}
& {[I \text { W }] \text { for } q \equiv 0(\bmod 3)} \\
& \text { [I qu] for } q \equiv I \text { or } 2(\bmod 3)
\end{aligned}
$$

over GF(3) Weighing codes. The purpose of tinis paper is to establish some general properties of weighing codes and to determine the welght distrioutions 
and desik: properties of the codes corresponding to $q=2$ and $a=3$.

Note that if

$$
G=[I \quad W]
$$

is the besis of $C$ then for $q \geq 1$ or $2(\bmod 3)$

$$
G^{l}=\left[\begin{array}{ll}
I & -W
\end{array}\right]
$$

is the basis of the dual code $C^{1}$. Hence $C$ is neither seif-dual nor selforthotonkl. Sowever we shall eee that $C$ and $C^{l}$ always nave tine sathe weight astribut ion and hence the same mininum distance d. By a well known result, cf. Delst.te [3], weighing codes are srthogonal arrays of strenth di-l. In this sense the weighing codes belong to a farlily of codes heludint the selfdubi code: see Mallows, et, al [7] and the symetry codes, see Pless [8, 9$]$ and Blaise [1].

We observe that the one's vector $\frac{1}{\sim} 3.5$ in $C$ for $q \equiv 1$ or 2 (mod 3) and $i \mathrm{~s}$ th, sum of the basis vectors. The vector $f=(1,1, \ldots, 1,-\ldots,-)$ (where represents -1 ) of $q^{2}+q+1$ ones and $q^{2}+q+1$ minuses oncurs In the dual cole for $q \equiv 1$ or 2 (mod 3 ).

If $q \equiv 0$ (mod 3) then the sum of the besis vectors

$$
\text { [I W] is not } \frac{1}{\sim} \text {, }
$$

anä so tise cole cannot contain $\frac{1}{\sim}$. Moreover, in this case rank $W<$ order of $W$ since $W^{2}{ }^{2} \equiv 0(\bmod 3)$.

2. GENETAA PRORERTIES OF TWE CODES.

If $A_{i} i s$ the number of codewords of weight $i$ in $C$, then we call the bivariate polynomial

$$
W E(x, y)=\sum_{i=0}^{n_{1}} A_{i} x^{n-i} y^{i}
$$

the wethit emmerator of $C$. If $A_{i j k}$ is the number of sodewords of weitht $s^{+} k$ in $\mathcal{C}$ containing $j$ ones and $j$ totos (minus ones over $G F(3)$ ) then we call the trivariate polynomial

$$
\operatorname{Cus}(x, y, z)=\sum_{i=0}^{n} A_{i j . j} x^{i} y^{j} z^{x}
$$

the comptete weight eniomeraton of $C$. 
THEOREM.

I.et $C$ be the code over $G F(q)$ with baeis $G=[I X]$ where $X$ is $a$ ciroulart matrix of order $k$ and $I$ is the identity matris of order $k$. Then $c$ and $C^{1}$ have the sane weight enwerators.

Proof :

First recall that if $X$ is airculant matrix and $R$ the back diagonal, permutation matrix then

$$
(X R)^{T}=X R \text {. }
$$

Now $C^{\perp}$ has basis

$$
\left[-x^{T} I\right]
$$

ard the basis vectors of $C^{1}$ may be written as

$$
R\left[-X^{T} I\right]=\left[-R X^{T} R\right]=\left[-X R^{T} R\right]=[-X R \quad R]
$$

since this mereiy involves rearranging the order of the basis vectors, Hence $C^{1}$ is equivalent to the code $D^{1}$ with basis

$[-X R$ I] as this just rearranges the columan of $R$. Since $X R$ is stmmetric we have that $\left(D^{\perp}\right)^{\perp}=D$ has basia $[I X R]$.

If $b$ is a q-ary vector of length $k$

then $\quad W E(Q[I X R])=W E(\underline{Q})+W E(\underline{b X R})$

whereas $\quad W E(b[-X R I])=W E(-b X R)+W E(b)$

and hence $D$ and $C^{\perp}$ have the same, weight enumerators, But $D$ is equivalent to $C$ and hence the theorem holds.

In particular $A_{i}=A_{1}^{\perp}$ for weighing codes, and so $C$ and $C^{\perp}$ form orthogonal arrays of maximum strength $d-1$ where $d$ is the minimum iistance of $C\left(\operatorname{ard} \mathfrak{C}^{\perp}\right)$.

Any two vectors from the basis of $C$ can be written as

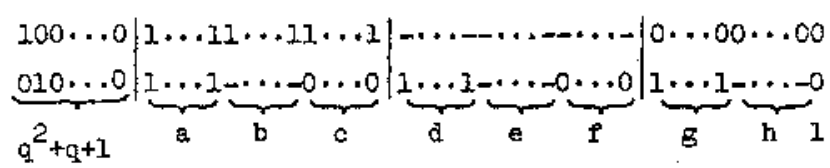

and we obtain the following equations

$$
\begin{aligned}
& a+b+c=a+d+b=\frac{1}{2}\left(q^{2}+q\right)=\text { number of ones. } \\
& a+e+f=b+e+b=\frac{1}{2}\left(q^{2}-q\right)=\text { number of minus ones. } \\
& 1+a+h=c+f+1=q+1=\text { number of zeros. } \\
& a+e=b+d \quad \text { (orthogonallty). }
\end{aligned}
$$


These equations can be silved for $c, d, e, f, g, h$ in terms of $q, a, t$. The CWE of the sum and difference of two vectors are

$$
x^{\frac{1}{2}}\left(3 q^{2}+q\right) y^{2+q^{2}+q-3 a} z-\frac{1}{2^{2}}{ }^{2}+\frac{1}{2} q+3 a
$$

and

$$
x^{\frac{1}{2}}\left(3 q^{2}+q\right) y^{1+q^{2}-3 b}-\frac{1}{2} q^{2}+\frac{3}{2} q+3 b+1
$$

respectirely.

of course the negatives of these vectors are also in $C$ and hence the weight of every two combinstion is $\frac{1}{2}\left(\alpha^{2}+3 q+4\right)$ and consequently there are at least $4\left(^{q^{2}}+q+1\right)$ vectors of this weight.

We moy observe that

$$
\frac{1}{2}\left(q^{2}+3 q+4\right)<q^{2}+1 \text { for } q \geq 4
$$

and henci $\frac{1}{2}\left(q^{2}+3 q+4\right)$ provides an upper bourd on the minimum distance of $C$ for $q \geq 4$.

3. TEE: : 14, 7) CODE WITH MIIIMUN DISTANCE 5.

\section{This cole is generated by w with first row}

$$
-110100
$$

In order to ensure the $\frac{1}{2}$ vector is in $\mathcal{C}$ we use the basis vectors

$$
G=\left[\begin{array}{ll}
I & 0
\end{array}\right]=[I-W]
$$

where $a=2$.

We observe that the lineer sombinations given by XO where $X=I+2+J \quad(Q$ as before the incidence matrix of the prosective plare of order 2 and $\left.W=Q^{2}-J\right)$ are

$$
\mathrm{H}=[\mathrm{X}-\mathrm{XW}]=[I+Q+J 2 Q+2 J](\bmod 3)
$$

and $K=2 H-3 J$ satiefies the equation $K K^{\mathrm{T}}: 16 \%$. $2 J$ over the real. numbers.

Since each rot of $K$ has eight $+I^{\prime} ;$ and six - l's and each colum: has fow + I's and three - I's we have a $(7,14,8,4,4)-$ BIBD. In fect the 16 vectors $\frac{1}{2}, 2, H, 2 H$ contair a $(14,16,6)$-block code. me vectors 


$$
\left[\begin{array}{lll}
1 & 1 & -I \\
\frac{T}{2} & \frac{1}{2} & -H
\end{array}\right],
$$

where I' $f$ s the vector of seven ones, are the flrst elght rows of an ladaramd matrix of order 16 (See Wallis, et al [11]).

We note that since every vector in the code $\mathfrak{C}$ is orthogonal to every tector in $\mathrm{C}^{1}$ the remaining 8 rows of this Hadamard matrix of order 16 (and their negatives) will be obtained from the vectors of full weight in $c^{1}$.

We found the weight distribution for this code, which is given in Figure 1, and that of the dual code, given in Figure 2. As expected, we see $C$ and $\mathcal{C}^{\perp}$ have the same welght distribution but not the same complete weight enumerator.

The (14,7)-code has minimum distance 5 and hence forms an orthogonal array of strength 4 .

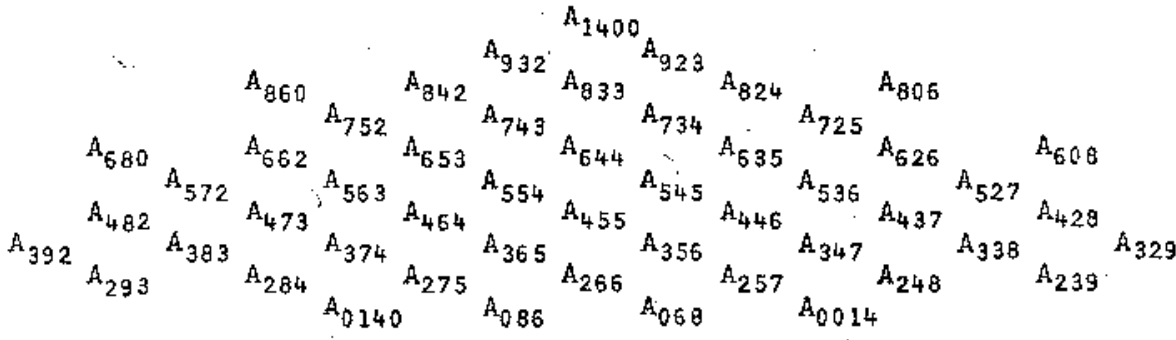

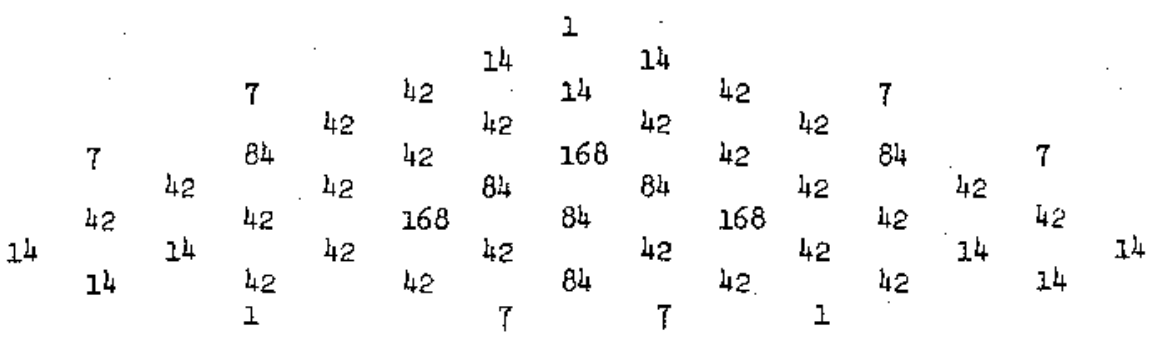




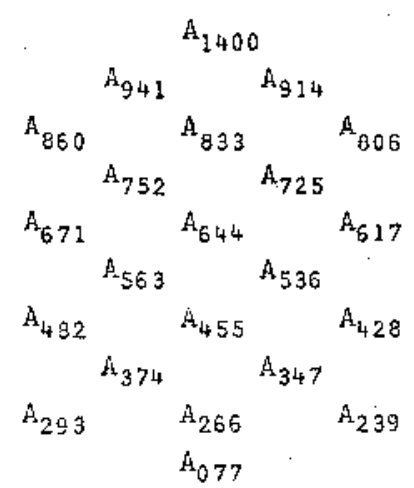

\begin{tabular}{|c|c|c|c|c|}
\hline & & $\mathbf{I}$ & & 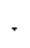 \\
\hline & 14 & & 14 & \\
\hline 7 & & 98 & & \\
\hline & 84 & & s. & \\
\hline 42 & & 350 & & 4 \\
\hline & .268 & & 168 & \\
\hline 84 & & 420 & & \&l. \\
\hline & 112 & & 212 & \\
\hline 55 & & 168 & & \\
\hline & & 16 & & \\
\hline
\end{tabular}

Figure 2.

4. TWO (26, 13)-CODES WIMH DISTANCE 3 AND 4

Richard M. Hain [5] conjectured ard Peter vades [4] verified (by conputer) that there are two equivalence classes of eirculant it $(13,9)$. They have first rows

$$
0-0-10011-112
$$

and

$$
0101100-11-1 .
$$

Call thu circuiant matrices with these sirst rows $w_{1}$ and $y_{2}$.

The linear codes $C_{1}, C_{2}$ with bases

$$
\left[\begin{array}{llll}
I & w_{1}
\end{array}\right],\left[\begin{array}{lll}
I & w_{2}
\end{array}\right]
$$

respectively, were studicd via the computer at the University of Sylney and theis CWE's outained. We give here their WE's in Firures 3 anc 4 respectively.

It is most interesting to note that the coles have different minimum distarces 3 and 4 rospectively. Also, di expected since $q=3 \equiv 0$ (nod 3) for these codes, neither $C_{1}$ nor $C_{2}$ contairs $\frac{1}{\sim}$ (and neither does $C_{1}^{\perp}$ nor $C_{2}^{\perp}$ as $I_{\text {I }}$ is not orthogonal to their basis vectors). Al: neither contains any full weight vectors.

Since the codes have minimum aistance 3 and 4 they are orthogonal arra: of stringth 2 and 3 respectively. 
$A_{0}=1$

$A_{1}=0$

$A_{2}=0$

$A_{3}=104$

$A_{4}=468$

$A_{5}=1404$

$A_{6}=4056$

$A_{7}=8424$

$A_{8}=11934$

$A_{9}=13442$

$A_{10}=11258$

$A_{11}=5928$

$A_{12}=4264$

$A_{13}=11260$

$A_{14}=39780$

$A_{15}=105768$

$A_{16}=211224$

$A_{17}=317538$

$A_{18}=352638$

$\mathrm{A}_{19}=281632$

$A_{20}=154128$

$A_{21}=52768$

$A_{22}=7904$

$\mathrm{A}_{23}=0$

$A_{24}=0$

$A_{25}=0$

$A_{26}=0$

Weight Distribution of $\mathcal{C}_{1}$

Figure 3 .
$A_{0}=1$

$A_{1}=0$

$A_{2}=0$

$A_{3}=0$

$A_{4}=26$

$A_{5}=0$

$A_{6}=156$

$A_{7}=624$

$A_{8}=0$

$A_{9}=1128$

$A_{10}=3458$

$A_{11}=8736$

$A_{12}=24830$

$A_{13}=54264$

$A_{14}=100152$

$A_{15}=152568$

$A_{16}=212862$

$A_{17}=259974$

$A_{18}=272766$

$A_{19}=222976$

$A_{20}=145002$

$A_{21}=73996$

$A_{22}=37180$

$A_{23}=16848$

$A_{24}=6006$

$A_{25}=780$

$A_{26}=0$

Weight Distribution of $\mathrm{C}_{2}$ Figure 4.

REESRENCES.

(1) Ian F. Blake, "On a generalization of the Fless symetry codes", Information and Control, 27(1975), 369-373.

(2) Ian F. Blake and honala C. Mullin, An Introduction to Algebraic and Combinatorial Coding theory, Academic Press, N.Y. -San Mrancisco-Iondon, 1976. 
(3) P. Delsarte, "Four Furdamental Parameters of a code and their cominator Significance", Infomation and Controt, 23(1973) $407-1$ it

(4) P. Eades, on the Existence of Orthogonal Designs, Ph.I. Fhesis, Aristral National University, Carberra, 2977.

(5) Richard M. Ha1n, Cinulant Weighing matrices, M.Sc. Thesis, Austraiian National Univeraity, Carberra, 1977.

(6) Marshall Hal. J.., Combinatorial Theory. Blaisdel1, [Ginn Co.], Waithar, Mass, 1967.

(7) C.I. Mallows, V. Pless and \$.J.A. Sloene, "Self-Dual codes ever GF(3)", SIAll J. Appl. Wath. Vol 3i, (1976), 649-666.

(8) V. Fless, "On a new farily of symetry codes and relsted new five desis. Buz2. Amer. Math. Soc. 75(1969), $1339-1342$.

(9) V. Pless, "Symmetry codes over GF(3) and now-five designs", J. Combinatorial Th. Ser. A 12(1972), 119-142.

(10) Jennifer Seberry Wallis and Albert Leon whiternan, "Sone results on weigh matrices", Bulz. Autral. Math. Soo. 12(1975), 433-447.

(11) W.D. Wallis, Anne Penfold Street, Jennifer Seberry Wallis, Combinatoric Roon Squares, sum-free sets, Hadanard matrices, jecture Hotes in Mathematics, Vol. 292, Springer-Verlag, Berlin-Heidelberg-New York, 1 : 